\title{
Timing and Duration of Gbx2 Expression Delineates Thalamocortical and Dopaminergic Medial Forebrain Bundle Circuitry
}

\author{
Elizabeth Normand1, Catherine Browning2, and Mark Zervas²*
}

1Department of Neuroscience and 2Department of Molecular Biology, Cell Biology and Biochemistry, Division of Biology and Medicine, Brown University, Providence, Rhode Island, USA

Note:

All research was planned and conducted while members were at Brown University

Email for correspondence:

MZ: Mark_Zervas@brown.edu

*Author for correspondence

\section{SUMMARY}

Gene expression is a dynamic process, which is highly coordinated during development to ensure the proper allocation and identity of neuronal cell types within the brain. Equally important during neurodevelopment is how cohorts of neurons establish axonal projections that innervate terminal target sites. We sought to bridge the temporal dynamics of gene expression, within a specific genetic lineage, to the establishment of neuronal circuits derived from cohorts of the lineage-specific progenitors. A central goal was to be able to accomplish genetic inducible circuit mapping non-invasively and with commonly available CreER/loxP technology. Specifically, we genetically marked thalamic neuron progenitors that expressed the transcription factor $G b \times 2$ at an early embryonic stage and tracked the formation of lineage-derived thalamocortical axons during embryogenesis. We then assessed the neural circuitry at an early postnatal stage. We show that the temporal specificity of lineage marking provides a high degree of clarity for following neural circuit development. We also determined that the onset and duration of gene expression can delineate subsets of neural circuits derived from a common lineage. For example, we uncovered a novel contribution of $G b x 2$-expressing progenitors to midbrain dopamine neurons and dopaminergic axons of the medial forebrain bundle. We anticipate that this system can be instructive in elucidating changes in neural circuit development in both normal development and in mutant mice in which neural circuit formation is altered.

\section{INTRODUCTION}

The relationship between a specific gene expressed in progenitors during development and the terminal cell fate of these progenitors is referred to as a genetic lineage. An important aspect of genetic lineage is how the timing of gene expression within the lineage helps shape the distribution and ultimate cell fate in mature tissues including the nervous system [1,2].

An important component of the genetic lineage of neurons and implicit in nervous system development is that axons derived from subsets of neurons anatomically bind discrete functional domains through the establishment of neural circuits. An important developmental problem is forging a link between progenitors that express genes with temporal precision and establishing neural circuits related to the lineagederived neurons. There have been recent advances in tackling the problem of neural circuit formation using mice as a model system (reviewed in [3] and see [4], but there are relatively few examples of marking neural circuits and establishing easily discernible point to point labeling of axonal connections based on temporal gene expression in mice. Thus, a desirable feature of circuitry mapping would be the use of a genetic-based system that can be used to mark progenitors with spatial and temporal control, conferring high fidelity of 
marking, and that could be readily used to better assess genetic mutant mice. It was previously shown that an inducible CreER recombinase-based system and a conditional GFP reporter allele allowed for the detection of lineage-derived axons [5,6]. More recently, a conditional red fluorescent reporter allele was used to mark/track thalamocortical circuits and the innervation of their target sites in control and mutant mice with fine spatial and temporal control [7].

Here, we use a well-characterized CreER line $[7,8]$ in combination with commercially available conditional reporter mice to characterize the distinct stages of axonal development of neurons derived from the Gbx2 lineage. Specifically, we marked and tracked this lineage with respect to developing thalamic neurons and their emerging circuits during in vivo development.

A surprising finding was that the Gbx2 lineage also contributed to midbrain dopamine neurons and to medial forebrain bundle circuitry. Collectively, these results reiterate that a genetics-approach is advantageous for elucidating temporal dynamics of gene expression and refining lineage-specific neural circuits in vivo. We anticipate that the ease and robustness of this approach will advance our understanding of neural circuit formation in normal development and in genetic mutant mice.

\section{MATERIALS AND METHODS}

\section{Mice and Fate Mapping}

Gbx2CreER mice were bred to $R 26^{\text {tdTomato }}$ reporter mice to generate $G b \times 2$ CreER-ires-eGFP;R26tdTomato mice that were subsequently bred to Swiss Webster female mice (Taconic) for genetic inducible fate mapping (GIFM) experiments. Gbx2CreER-ires-eGFP mice [8] were generously provided by James Li ( $\mathrm{UCHC}$ ) while the Rosa26tdTomato (Rosa26lox-STOP-lox-tdTomato) [9] line was purchased from Jackson Laboratories.

Adult Gbx2CreER-ires-eGFP;R26 $6^{\text {tdTomato }}$ male mice between four and six weeks of age were set up in breeding pairs with Swiss Webster (Taconic) females and were checked for the presence of a vaginal plug each morning at 0900 hours. The presence of a plug indicated a successful breeding event and was considered 0.5 days post conceptus or embryonic day (E)0.5. Pregnant female mice harboring embryos at E9.5 were administered $4 \mathrm{mg}$ $(200 \mu \mathrm{l})$ of tamoxifen (T-5648, Sigma) from a stock solution of tamoxifen in corn oil $(20 \mathrm{mg} / \mathrm{ml})$ at 0900 hours by oral gavage as previously described $[5,10,11]$. Litters were then dissected at E12.5 or E18.5. An additional cohort of mice that were allowed to go to term were sacrificed at postnatal day $(P) 7$. All mice were housed, handled, and euthanized in accordance with IACUC guidelines at Brown University.

\section{Tissue Processing}

Embryos were dissected in PBS over ice and a small tail biopsy was used for genotyping. P7 mice were perfused with $4 \%$ paraformaldehyde (PFA)/saline and brains were removed as previously described $[5,10]$. Embryos and brains were fixed in PFA overnight at $4{ }^{\circ} \mathrm{C}$. Subsequently, tissue was rinsed in PBS, immersed in $15 \%$ sucrose, and $30 \%$ sucrose until fully submerged. Tissues were embedded in Optimal Cutting Iemperature (OCT) media in cryomolds. The OCT/cryomolds were then immersed in a polypropylene beaker containing 2methyl-butane that was immersed in a vessel containing liquid nitrogen until the temperature reached $-150^{\circ} \mathrm{C}$ as previously described [10]. Sections were obtained with a Leica cryostat and mounted on slides.

\section{Immunocytochemistry (ICC) and microscopy}

Sections $(12 \mu \mathrm{m})$ were rinsed in PBS for 5 minutes (min) and fixed in 4\% PFA in PBS for 5 min. Slides were then rinsed 3 times in $0.2 \%$ TritonX-100 in PBS (PBT) for 5 min each and blocked in $10 \%$ donkey serum in PBT for 2 hours (h) at room temperature in a humid box.

Sections were immunolabeled using an anti-DsRed antibody that detects the protein product generated by the recombined $R 26^{\text {tdTomato }}$ reporter allele (anti-dsRed $\mathrm{Ab}$ from Clontech, Catalog (Cat) \#632496, 1:500 in $10 \%$ donkey serum in PBT). We also used an anti-TH primary antibody (Chemicon; Billerica, MA; Cat \#AB152, 1:500 in 10\% donkey serum in PBT) to detect dopamine neurons and their axons at E12.5. Appropriate, species matched Alexa secondary antibodies (Molecular Probes) were prepared at a concentration of $1: 500$ in $1 \%$ donkey serum in PBT. Sections were incubated in $300 \mu$ l of secondary antibody solution for $2 \mathrm{~h}$ at room temperature, washed with PBT five times for $10 \mathrm{~min}$ each, and counterstained with $.01 \%$ Hoechst 33342 (Molecular Probes; Cat \# H-3570) in PBS for 5 min in the dark. Slides were washed two times with PBS for 2 min each, dried and coverslipped.

Low magnification images (Figures 1A, 3A, and 4A) were captured with a Leica MZ16F stereo fluorescent dissecting microscope using PictureFrame software. High magnification images images were collected with a Leica DM6000B epifluorescent microscope using Volocity 5.1 imaging software (Improvision) and were obtained using a motorized stage with a 20x objective. True magnifications are indicated by scale bars in figures. All images were pseudo-colored live as part of the acquisition palette and protocol. Subsequently, imaging data sets were exported to Adobe Photoshop and montages of representative data were generated and presented here. 


\section{RESULTS}

We administered tamoxifen to E9.5 Gbx2CreER-ires-eGFP embryos to mark thalamic progenitors in vivo $[7,8]$. We coupled this mouse line with R26tdTomato conditional reporter mice because they provide a robust readout of Cre-mediated recombination [7,9]. In the absence of tamoxifen, CreER protein is sequestered in the cytoplasm and recombination of conditional alleles does not occur $[5,11]$. However, delivering tamoxifen to pregnant female mice (see Methods) controls the temporal release of CreER from sequestration, which frees it to translocate to the nucleus where it mediates recombination between same-site oriented loxP sites that flank a stop cassette in the reporter allele (reviewed in [2]). Thus, tamoxifen administration allows us to control the timing of the recombination event and cell marking within the Gbx2 lineage.

\section{Gbx2 derived neural circuit formation}

Subsequent to tamoxifen administration at E9.5, we analyzed embryos at mid-gestation (E12.5) of development, which revealed thalamic neurons that were derived from the Gbx2 lineage (Figure 1A,B). The marked neurons had axons that emerged from the rostral thalamus and formed a thick proximal fascicle, which continued to project rostrally (Figure 1A-C).
Gbx2-derived axonal projections terminated at a distal limit in the proximity of the thalamocortical intermediate target zone (Figure 1D) consistent with thalamocortical axon guidance [12]. A second cohort of Gbx2-derived neurons were located in the ganglionic eminence (Figure 1A,E). In addition to thalamocortical projections, there was a more ventrally located, loose axonal fiber tract in what appeared to be the medial forebrain bundle (MFB, Figure 1A, white arrow).

\section{Dynamic gene expression delineates neural circuits}

The Gbx2CreER-ires-eGFP;R26tdTomato allelic configuration allows for the determination of progenitors that expressed Gbx2 at the stage of marking (E9.5, red) and whether they continued to express Gbx2 at the stage of analysis (E12.5, green). We previously found this approach to be instructive in determining how the timing and duration of Gbx2 expression shaped spinal cord development [13]. In this study, we assessed dynamic gene expression and early thalamic circuits using lineage analysis and identified neural circuits that were derived from early $G b x 2$ expressing progenitors marked at E9.5 (tdTomato+, red) and neurons that continued to express Gbx2(GFP) at the time of analysis (GFP+ neurons and their axons, green). Interestingly, the

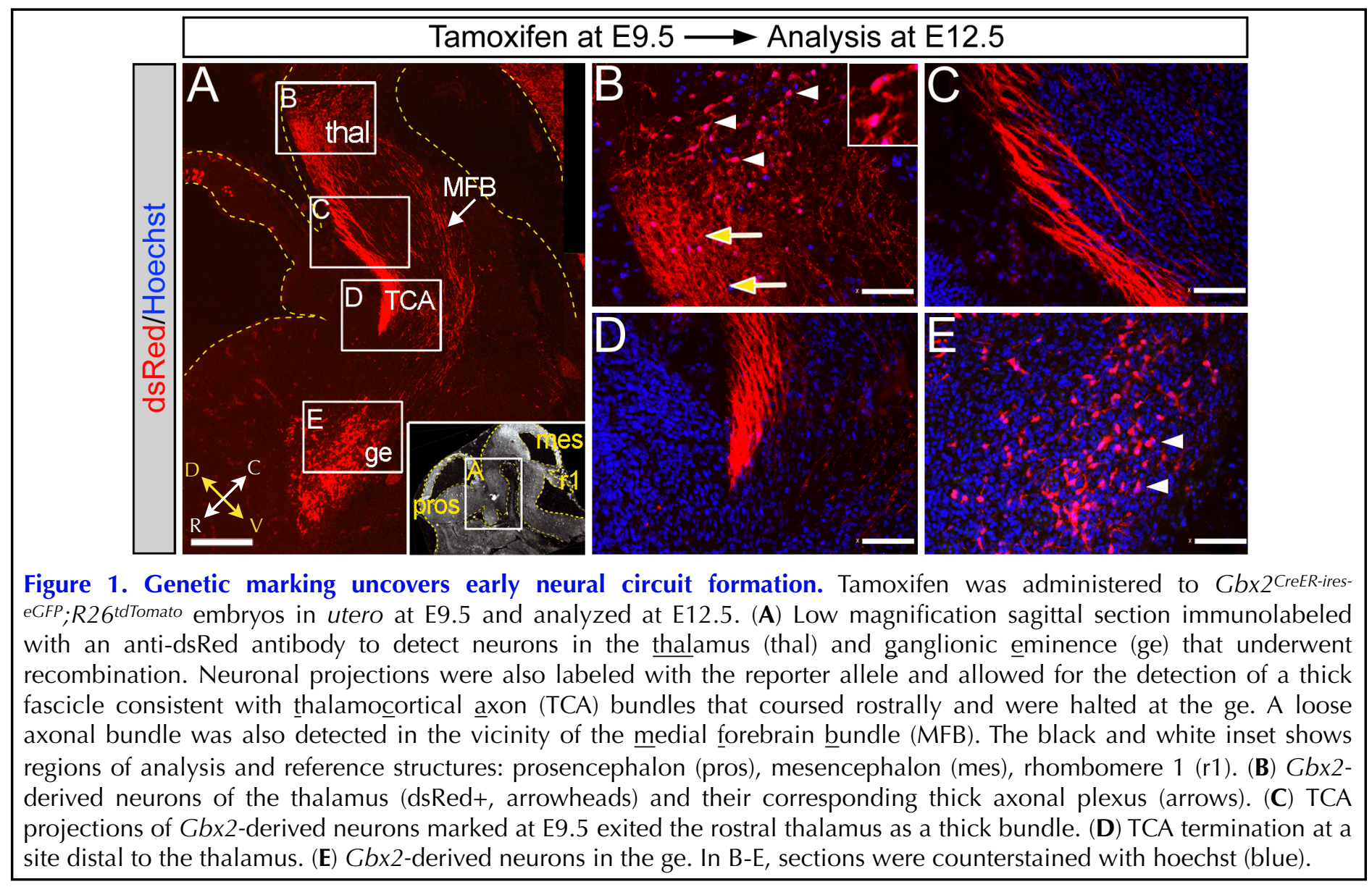




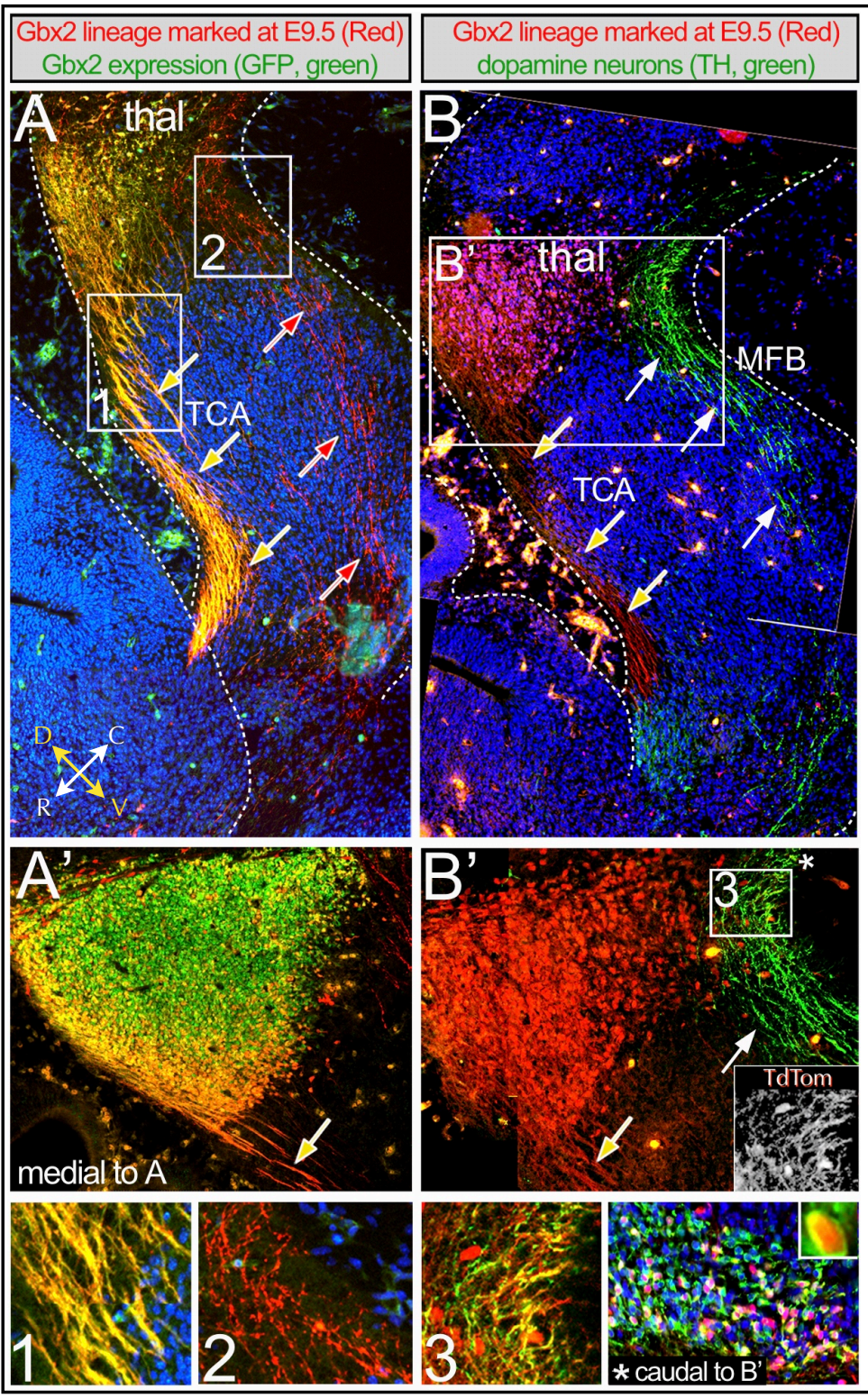

Figure 2. Timing of gene expression defines subsets of neural circuits within a defined genetic lineage. Tamoxifen was administered to Gbx2CreER-ires-eGFP;R26tdTomato embryos at E9.5 and analyzed at E12.5. (A) Sagittal section immunolabeled with anti-dsRed and anti-GFP antibodies to detect thalamic neurons that, respectively, expressed Gbx2 at E9.5 (dsRed+, red) and continued to express Gbx2 at E12.5 (GFP+, green). Sections were counterstained with hoechst (blue). Neurons in the thalamus (thal) and TCA (yellow arrows) were dsRed+/GFP+ (yellow) indicating that they were derived from neuronal progenitors that expressed Gbx2 at E9.5 and continued to express Gbx2 at E12.5. The axons of the medial forebrain bundle (MFB) were dsRed+/ GFP- (red arrows) indicating that these projections were related to neurons that expressed Gbx2 early, but ceased

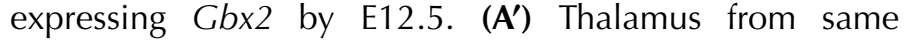
sample but at more medial location. (1,2) High magnification panels of regions of interest shown in A. (B) Sagittal section immunolabeled with anti-dsRed and antityrosine hydroxylase (TH) antibodies to detect neurons that expressed Gbx2 at E9.5 (dsRed+, red) and to identify dopamine neurons ( $\mathrm{TH}+$, green), respectively. Neurons in the thal and TCA were dsRed+ consistent with adjacent section in A. The MFB contained TH+ dopaminergic axons (white arrows). (B') Thalamic and dopaminergic axons coursing ventral to the thal. The $\mathrm{TH}+$ projections were also dsRed+ but were much fainter than the neurons in the thalamus. Black and white inset shows red channel alone from region 3. (3) High magnification panel of region 3 with the red signal increased to observe the projections. The asterisk $\left(^{*}\right)$ in $\mathrm{B}^{\prime}$ is a reference for the domain caudal and medial to panels shown in $B, \mathrm{~B}^{\prime}$ at the ventral mesencephalic flexure. The domain is depicted in the panel [*caudal to $\left.\mathrm{B}^{\prime}\right]$ and shows midbrain dopamine neurons derived from the Gbx2 lineage marked at E9.5 (dsRed+/TH+, yellow) that project rostrally via the MFB. thalamocortical axons (TCA) at E12.5 were derived from neurons that had both early and persistent $G b \times 2$ expression (tdTomato+/GFP+, yellow) (Figure 2A, $\mathbf{A}^{\prime}$ ). In contrast, the Gbx2-derived axons of the putative MFB did not continue to express Gbx2(GFP) (Figure 2A). We then used antibody labeling to detect tyrosine hydroxylase $(\mathrm{TH})$ which labels midbrain dopamine neurons and $\mathrm{TH}+$ axons that course along the MFB $[14,15]$. Surprisingly, Gbx2-derived axons (dsRed+) in the MFB were also $\mathrm{TH}+$, which suggests that midbrain dopamine neurons were derived from Gbx2-derived progenitors marked at E9.5 (Figure $\mathbf{2 B}, \mathbf{B}^{\prime}$ ). Therefore, we investigated whether midbrain dopamine neurons, are directly derived from Gbx2-expressing progenitors.

It was notable that co-immunolabelling with $\mathrm{TH}$ and dsRed antibodies validated that some midbrain dopamine neurons $(\mathrm{TH}+)$ were derived from the $G b \times 2$ lineage $(\mathrm{dsRed}+)$. During embryogenesis Gbx2expressing progenitors are positioned caudal to the ventral mesencephalon and located in the ventral domain of rhombomere 1 [16]. It was previously shown that a lineage restriction boundary is established between the ventral domains of the mesencephalon and r1 shortly after E9.5 [17], which prevents midbrain progenitors from contributing to r1-derived structures. However, these findings suggest that the boundary is not bi-directional and that some ventral $r 1$ progenitors expressing Gbx2 traverse this (mesencephalic) lineage boundary and contribute to midbrain dopamine neurons (Figure $\mathbf{2} \mathbf{B}^{\prime}$ and the inset *audal to $\mathbf{B}^{\prime}$ ). Thus, both TCA and MFB circuits are derived from the Gbx2 lineage at E9.5, but the TCA bundle persists in expressing Gbx2 at least until E12.5 while the MFB only transiently expresses Gbx2, which is ceased by E12.5.

\section{Maturation of thalamocortical circuits}

The Gbx2 lineage-derived neurons marked at E9.5 continued to populate the thalamus at E18.5 (Figure 
3A,B). These thalamic neurons had axons that exited the ventral aspect of thalamus (Figure $\mathbf{3 A}, \mathbf{C}$ ) and traversed ventrally and rostrally as fascicles, which passed through the ventral half of the striatum (Figure 3A,D). The bundles de-fasciculated as they entered the cerebral cortex and formed fine axonal branches, which traversed the deep cortical layers (Figure 3A,E). In addition, tdTomato (dsRed+) projections were used to follow entire axonal bundles oriented in parallel to each other en route to the deep cortical layers in more lateral sections, which revealed robust innervation of layer 6 (Figure 3F). Projections proximal to layer 6 were dense (in deep layers), but without obvious orientation. The marked projections became much sparser in more superficial cortical layers (Figure 3F).

\section{Early postnatal thalamocortical circuits}

Finally, thalamic neurons derived from Gbx2expressing progenitors marked at E9.5 were distributed broadly throughout the thalamus by the first postnatal week (P7) (Figure 4A,B). These neurons had characteristic thalamic morphologies (Figure 4B, inset). In addition, thalamic neurons had broad sweeping fascicles that apparently emerged from the rostral-lateral extent of the thalamus and entered the internal capsule (Figure 4A,C).

The fascicles remained well organized and appeared as parallel-oriented fibers with numerous small terminal ramifications throughout and at the anteriordorsal extent of the caudate/putamen (Figure 4A,D). Significant cohorts of thalamocortical projections passed through the internal capsule and caudate/ putamen as smaller bundles when compared to their entrance into the internal capsule. These Gbx2-derived projections retained a parallel orientation with each other as they entered into layer $6 \mathrm{~b}$ of the cerebral cortex (Figure 4A,E,F).

Gbx2-derived neurons were also located ventral and rostral to the thalamus (Figure $\mathbf{4 A}, \mathbf{G}$ ). In adjacent parallel sections (Figure $\mathbf{4} \mathbf{H}, \mathbf{I}$ ) the $G b \times 2$-derived axons innervated somatosensory cortex resulting in the coalesced innervation of the immature somatosensory barrels of cortical layer 4 (Figure $\mathbf{4 H}$, inset). In addition, deep-layer projections were organized as a dense fiber tract with long, longitudinal processes orientated along the rostral-caudal axis within layer $6 \mathrm{~b}$ (Figure 4I). In summary, aside from somatosensory innervation, once thalamocortical axons innervated the cerebral cortex, these fibers de-fasciculated and formed numerous fine ramifications in the cortex. However, in somatosensory cortex, Gbx2-derived projections formed dense axonal clusters and established the rudimentary somatosensory barrels.

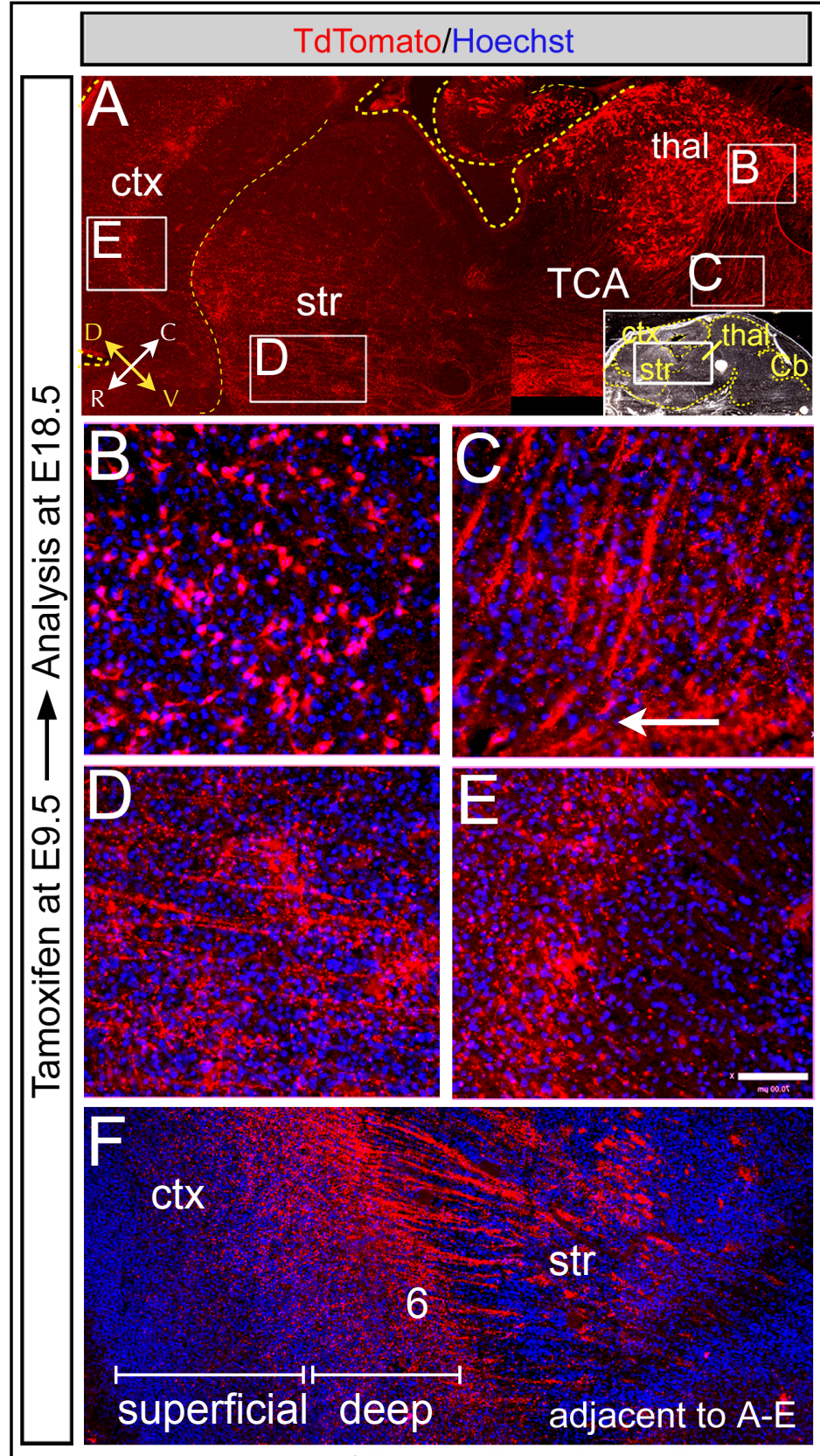

Figure 3. Maturation of lineage derived neural circuits. Tamoxifen was administered to Gbx2CreER-ires-eGFP;R26tdTomato embryos at E9.5 and analyzed at E18.5. (A) Sagittal section immunolabeled with an anti-dsRed antibody to detect neurons in the thalamus (thal) that underwent recombination. Sections were counterstained with hoechst (blue). The TCA bundle traversed the striatum (str) and entered the deep layers of the cerebral cortex (ctx). (B) Thalamic neurons that expressed Gbx2 at E9.5 were becoming morphologically distinct. (C) The TCA fascicles exited the thal as thick well-defined processes that turned rostrally (arrow). (D) The Gbx2 derived projections entered the striatum and formed a fine network of axon collaterals , which was in contrast to TCA marked at the same stage, but analyzed at E12.5 (See Figure 1). (E) TCA that exited the striatum ramified the the deep cortical layer. (F) In a section adjacent to panels A-E, Gbx2-derived TCA passed through the striatum and innervated deep cortical layers with fine axonal branches that were progressively sparser as they ramified layers more superficial to cortical layer 6 . 


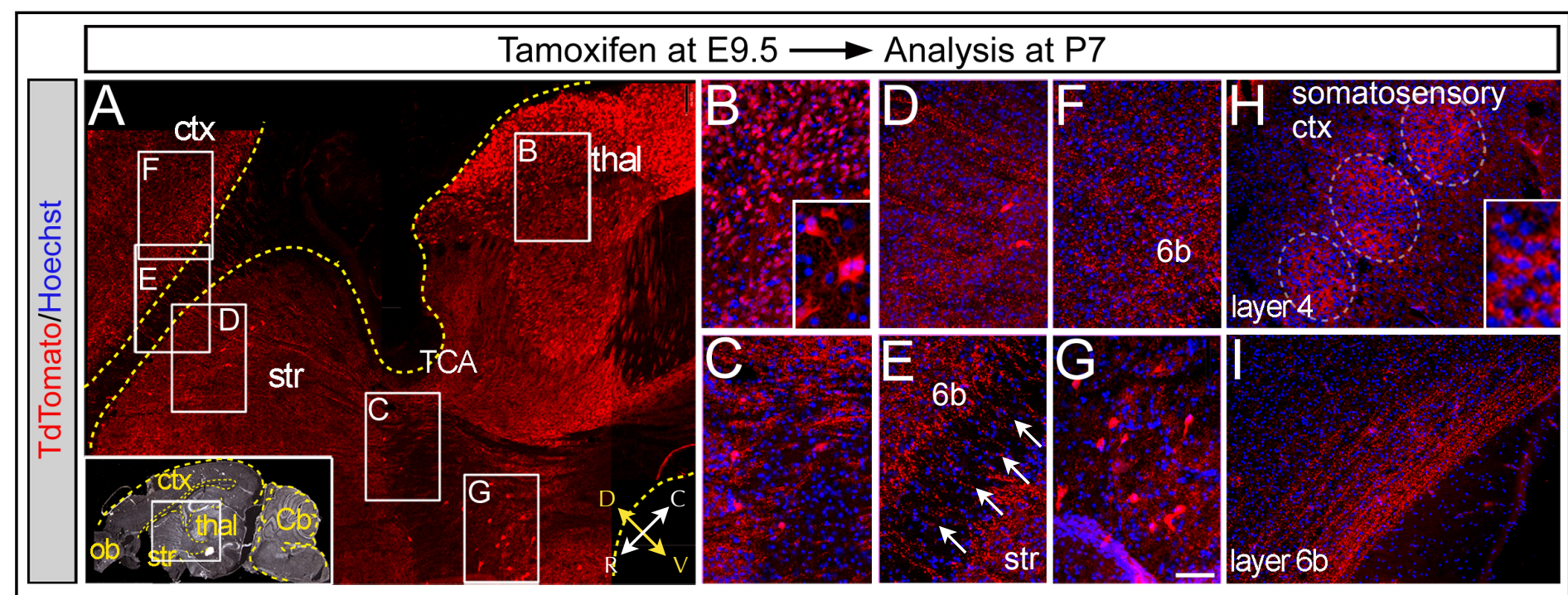

Figure 4. Target innervation of genetic lineage derived neural circuits. Tamoxifen was administered to Gbx2CreER-ireseGFP;R26tdTomato embryos at E9.5 and analyzed at P7. (A) Sagittal section immunolabeled with an anti-dsRed antibody detected neurons and the thalamocortical axon bundle (TCA) derived from the Gbx2 lineage marked at E9.5. Sections were counterstained with hoechst (blue). Gbx2-derived TCA traversed the striatum (str) as thick bundles where they branched and formed fine axonal terminations. The TCA exited striatum and innervated the cerebral cortex (ctx). (B) Neuronal progenitors that expressed Gbx2 at E9.5 contributed to mature thalamic neurons that were distributed in the thal. (C) Numerous thick TCA bundles exited the thal. (D) TCA traversed the entire distance of the str as thick bundles with fine axonal branches that established a Gbx2-derived plexus in the caudate/putamen of the str. $(\mathbf{E}, \mathbf{F})$. Upon exiting the str, the TCA projections were thinner in comparison to when they entered and traversed the str. The TCA then formed a dense plexus in deep cortical layer 6b. $(\mathbf{G})$ Gbx2-derived neurons remaining in close proximity, but ventral to the thal. $(\mathbf{H}, \mathbf{I})$. In an adjacent sagittal sections to A-G, Gbx2-derived axons innervated somatosensory cortex where the axons coalesced in a fingerprint pattern, consistent with the early formation of barrel cortex $(\mathrm{H})$. In addition, Gbx2-derived axons traversed the rostral-caudal axis as long axonal bundles that were oriented parallel to the ventral surface (I).

\section{DISCUSSION}

The thalamus is comprised of both locally projecting interneurons and long-distance projection neurons [18]. Physical tracing methods have been instructive in elucidating the formation of thalamocortical axons and is a well-described procedure that shows that thalamic axons emanate from the thalamus and halts at intermediate target sites. Subsequently, thalamic axons turn toward and innervate the cerebral cortex $[12,19,20,21]$. It has been shown that the Gbx2 lineage contributes to thalamic neurons and that $G b \times 2$ is required for the proper formation of thalamic axonal projections $[7,22,23]$. In addition, $G b \times 2$ is required cell non-autonomously for thalamic development [8].

However, the methods used in the aforementioned thalamocortical circuitry studies have been invasive and, therefore, may have labelled fibers of passage. Additionally, a relationship between the timing of gene expression, genetic lineage, and neural circuit formation has not been established for the Gbx2 lineage. Thus, we sought to use GIFM as an approach to demonstrate whether the early expression of Gbx2 in thalamic neuron progenitors was related to the formation of long distance axonal circuits that eventually innervate cortical targets. We marked Gbx2 expressing progenitors at E9.5 and analyzed early patterns of axonal growth after three days. We observed clearly labeled Gbx2 lineage-derived thalamocortical axons that exited the thalamus in thick fascicles and were abrogated at the ganglionic eminence consistent with previous physical axonal tracing methods [18]. By E18.5 Gbx2 derived axons entered the striatum as thick fascicles and formed a rich axonal plexus. Upon exiting the caudate/putamen, well-defined axonal tracts entered the deep cortical layers with fine axons that began to ramify into more superficial layers. At P7, the axons that entered the cortex formed an axonal tract that traversed the rostral-caudal axis of the cortex. Notably, at E18.5 only fine axons innervated more superficial cortical layers, while at P7 the projections establish a broad zone of termination in layer 4 of cortex. In the somatosensory cortex, the projections coalesced as the rudimentary layer 4 barrel structures.

During the course of our analysis, we also observed a second, surprising Gbx2 lineage-derived axonal tract. This second bundle was located ventrally to the thalamus as part of the putative medial forebrain bundle. Thus, we show for the first time that a portion of medial forebrain bundle axons are derived from Gbx2- 
expressing progenitors that contribute to dopamine neurons. It is well known that Gbx2 expression defines a domain that is posterior to the germinal zone of ventral midbrain progenitors $[24,25,26,27]$. However, a recent study showed that Gbx2 transcript is expressed in the ventral mesencephalic midline at E10.5 and that Gbx2 lineage contribution occurs in the ventral-medial midbrain primordia [28]. This ventral-medial primordia is the in vivo source of midbrain dopamine neurons $[29,30]$, which are connected to the cerebral cortex via the medial forebrain bundle. However, the aforementioned study [28] did not demonstrate a direct link between Gbx2-expressing progenitors and terminally fated midbrain dopamine neurons and dopaminergic circuitry. It is quite likely that a portion of the dopaminergic projections that were derived from the Gbx2 lineage are part of the nigrostriatal bundle in addition to the medial forebrain bundle.

We show here that thalamocortical axons were derived from neuronal progenitors that had early (E9.5) and persistent (E12.5) expression of Gbx2 (tdTomato+/GFP+, yellow). In contrast, dopaminergic axons of the medial forebrain bundle (and their related dopamine neurons) were derived from progenitors that expressed Gbx2 only early and transiently (at E9.5, but not at E12.5).

In summary, this genetic inducible circuit mapping approach makes it possible to link the differential timing of gene expression and specific neural circuits within a unique genetic lineage. Finally, we show that a conditional genetic-based approach is highly effective at marking and following neural circuit formation and maturation in vivo. This genetic inducible circuit mapping approach has distinct advantages over traditional methods including that it is non-invasive and is highly reproducible. In addition, there is no confounding labeling of fibers of passage. In conclusion, this combination of GIFM and circuit tracing forges a link between temporal gene expression during embryogenesis and postnatal neural circuits.

\section{ACKNOWLEDGEMENTS}

This work was supported by a Department of Defense CDMRP grant (TS110067, MZ) and the Brown University Department of Neuroscience training grant (NS062443-02).

\section{REFERENCES}

1. Dymecki, SM and Kim, JC (2007). Molecular neuroanatomy's "Three Gs": a primer. Neuron 54, 17-34.

2. Joyner, AL and Zervas, M (2006). Genetic inducible fate mapping in mouse: Establishing genetic lineages and defining genetic neuroanatomy in the nervous system. Dev. Dyn. 235, 2376-2385.
3. Luo, L, Callaway, EM, and Svoboda, K (2008). Genetic dissection of neural circuits. Neuron 57, 634-660.

4. Lo, L, and Anderson, DJ (2011). A cre-dependent, anterograde transsynaptic viral tracer for mapping output pathways of genetically marked neurons. Neuron 72, 938-950.

5. Ellisor, D, Koveal, D, Hagan, N, Brown, A, and Zervas, M (2009). Comparative analysis of conditional reporter alleles in the developing embryo and embryonic nervous system. Gene Expr. Patterns 9, 475-489.

6. Hagan, N and Zervas, M (2011). Wnt1 expression temporally allocates upper rhombic lip progenitors and defines their terminal cell fate in the cerebellum. Mol. Cell Neurosci. 49, 217-229.

7. Normand EA, Crandall SR, Thorn CA, Murphy EM, Voelcker B, Browning C, Machan JT, Moore Cl, Connors BW, Zervas M (2013). Temporal and mosaic TsC1 deletion in the developing thalamus disrupts thalamocortical circuitry, neural function, and behavior. Neuron 78, 895-909.

8. Chen, L, Guo, Q, and Li, JY (2009). Transcription factor Gbx2 acts cell-nonautonomously to regulate the formation of lineage-restriction boundaries of the thalamus. Development 136, 1317-1326.

9. Madisen, L, Zwingman, TA, Sunkin, SM, Oh, SW, Zariwala, HA, Gu, H, Ng, LL, Palmiter, RD, Hawrylycz, MJ, Jones, AR, Lein, ES, and Zeng, H (2010). A robust and high-throughput Cre reporting and characterization system for the whole mouse brain. Nat. Neurosci. 13, 133-140.

10. Brown, A, Brown, S, Ellisor, D, Hagan, N, Normand, E, and Zervas, $M$ (2009). A practical approach to genetic inducible fate mapping: a visual guide to mark and track cells in vivo. J. Vis. Exp., pii: 1687.

11. Ellisor, D and Zervas, M (2010). Tamoxifen dose response and conditional cell marking: Is there control? Mol. Cell Neurosci. 45, 132-138.

12. Inan, M and Crair, MC (2007). Development of cortical maps: perspectives from the barrel cortex. Neuroscientist 13, 49-61.

13. Luu, B, Ellisor, D, and Zervas, M (2011). The lineage contribution and role of $G b x 2$ in spinal cord development. PLoS One 6, e20940.

14. Blakely BD, Bye CR, Fernando CV, Horne MK, Macheda ML, Stacker SA, Arenas E, Parish CL (2011). Wnt5a regulates midbrain dopaminergic axon growth and guidance. PLoS One 6: e18373.

15. Vitalis, T, Cases, O, Engelkamp, D, Verney, C, and Price, DJ (2000). Defect of tyrosine hydroxylaseimmunoreactive neurons in the brains of mice lacking the transcription factor Pax6. J. Neurosci. 20, 6501-6516. 
bioRxiv preprint doi: https://doi org/10.1101/579664; this version posted March 15, 2019. The copyright holder for this preprint (which was not certified by peer review) is the author/funder, who has granted bioRxiv a license to display the preprint in perpetuity. It is made available under aCC-BY-NC-ND 4.0 International license.

16. Ellisor D, Rieser C, Voelcker B, Machan JT, Zervas M (2012). Genetic dissection of midbrain dopamine neuron development in vivo. Dev. Biol. 372, 249-262.

17. Zervas, M, Millet, S, Ahn, S, Joyner, AL (2004). Cell behaviors and genetic lineages of the mesencephalon and rhombomere 1 . Neuron 43, 345-357.

18. Jones, EG (2007). The Thalamus. Second edition, volume I and volume II. Cambridge University Press. Published in the USA by Cambridge University Press, New York. www.cambridge.org/9780521858816

19. Bayer, SA and Altman, J (1991). Neocortical Development. Raven Press Ltd., New York, NY.

20. Adams, NC and Baker, GE (1995). Cells of the perireticular nucleus project to the developing neocortex of the rat. J. Comp. Neurol. 359, 613-626.

21. Vanderhaeghen, P and Polleux, F (2004). Developmental mechanisms patterning thalamocortical projections: intrinsic, extrinsic and in between. Trends Neurosci. 27, 384-391.

22. Hevner, RF, Miyashita-Lin, E, and Rubenstein, JL (2002). Cortical and thalamic axon pathfinding defects in Tbr1, Gbx2, and Pax6 mutant mice: evidence that cortical and thalamic axons interact and guide each other. J. Comp. Neurol. 447, 8-17.

23. Miyashita-Lin, EM, Hevner, R, Wassarman, KM, Martinez, S, and Rubenstein, JL (1999). Early neocortical regionalization in the absence of thalamic innervation. Science 285, 906-909.

24. Wassarman, KM, Lewandoski, M, Campbell, K, Joyner, AL, Rubenstein, JL, Martinez, S, and Martin, GR (1997). Specification of the anterior hindbrain and establishment of a normal mid/hindbrain organizer is dependent on Gbx2 gene function. Development 124, 2923-2934.

25. Martinez-Barbera, JP, Signore, M, Boyl, PP, Puelles, E, Acampora, D, Gogoi, R, Schubert, F, Lumsden, A, and Simeone, A (2001). Regionalisation of anterior neuroectoderm and its competence in responding to forebrain and midbrain inducing activities depend on mutual antagonism between OTX2 and GBX2. Development 128, 4789-4800.

26. Li, JY and Joyner, AL (2001). Otx2 and Gbx2 are required for refinement and not induction of mid- hindbrain gene expression. Development 128, 4979-491.

27. Li, JY, Lao, Z, and Joyner, AL (2002). Changing requirements for $G b \times 2$ in development of the cerebellum and maintenance of the mid/hindbrain organizer. Neuron $36,31-43$.

28. Sunmonu, NA, Li, K, Guo, Q, and Li, JY (2011). Gbx2 and Fgf8 are sequentially required for formation of the midbrain-hindbrain compartment boundary. Development 138, 725-734.
29. Brown, A, Machan, JT, Hayes, L, and Zervas, M (2011). Molecular organization and timing of Wnt1 expression define cohorts of midbrain dopamine neuron progenitors in vivo. J. Comp. Neurol. 519, 2978-3000.

30. Hayes L, Zhang Z, Albert P, Zervas M, Ahn S (2011). Timing of sonic hedgehog and Gli1 expression segregates midbrain dopamine neurons. J. Comp. Neurol. 519, 3001-3018. 